\title{
Twisting of the Tail Rotor Shaft - A Case Study MUHAMMAD Shahzad ${ }^{\mathrm{a},{ }^{*},}$, TANVEER Manzoor ${ }^{\mathrm{b}}$, QANITA Tayyaba $^{\mathrm{c}}$, AMMAD Hussain Qureshi ${ }^{d}$ \\ Materials Division, PINSTECH, P.O. Nilore, Islamabad, Pakistan \\ ashahzad@live.de, btanvirmanzoor@yahoo.com, 'qanita750@yahoo.com, dammad@pinstech.org.pk
}

\section{Keywords: Rotor Shaft, Strain Rate, Mechanical Behavior, ANSYS Simulation}

\begin{abstract}
Presented results report the findings of a case study carried out to determine the possible factors that lead to the twisting of tail rotor shaft. The structural material of the shaft was evaluated in terms of microstructural analysis and mechanical properties to rule out any material fault. The SEM images showed that the localized fractures at twist ends occurred without any significant plastic deformation. Moreover, there was no evidence of fatigue. Such behavior suggests that twist occurred under impact / high strain rate loading. Such loading conditions are not possible during the event to ground hitting. The Ansys simulation confirmed that the observed twisting can increase the stress at localized point in excess of UTS and cause fracture.
\end{abstract}

\section{Introduction}

Role of Tail Rotor Torque. As per Newton's third law of motion, every action has a reaction. The high speed rotation of overhead rotor blades which is vital to provide the lift off, however the same rotation produces a torque in the direction, which if not countered would force the helicopter to spin around its axis in the direction opposite to the rotating blades. As shown in Fig. 1, the anti-clock rotation of overhead rotor blades produces a clockwise torque which is being countered by the tail rotor blades to keep the helicopter stable and straight. In addition, the rotor blades are used to change the direction of the helicopter by changing the pitch (angle of attack) of the blades.

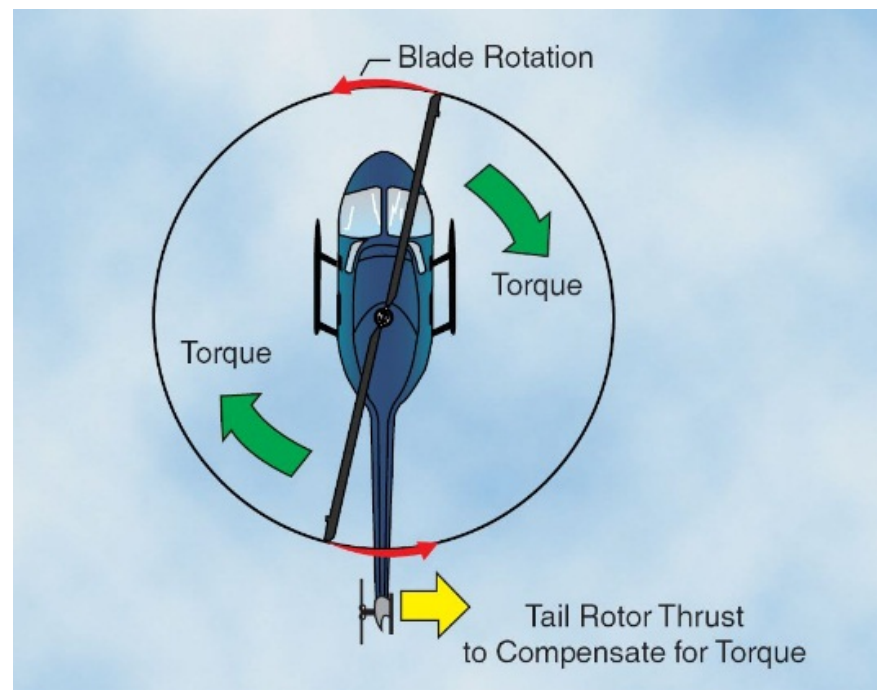

Fig. 1. Counter torque by the tail rotor shaft [1]

Description of Event. A helicopter under a routine field operation was lifting off. It successfully lifted off and attained some good height. However, as per the information provided, within seconds, it came down while spinning and hit the ground from the right side.

One of the observation made during the detailed visual inspection was that the tail rotor shaft of the helicopter was twisted and has become loose probably due to the consequent shortening of the twisted shaft (Fig. 2a). The loose rotating shaft caused some damage to the outer casing at several places (Fig. 2b). 

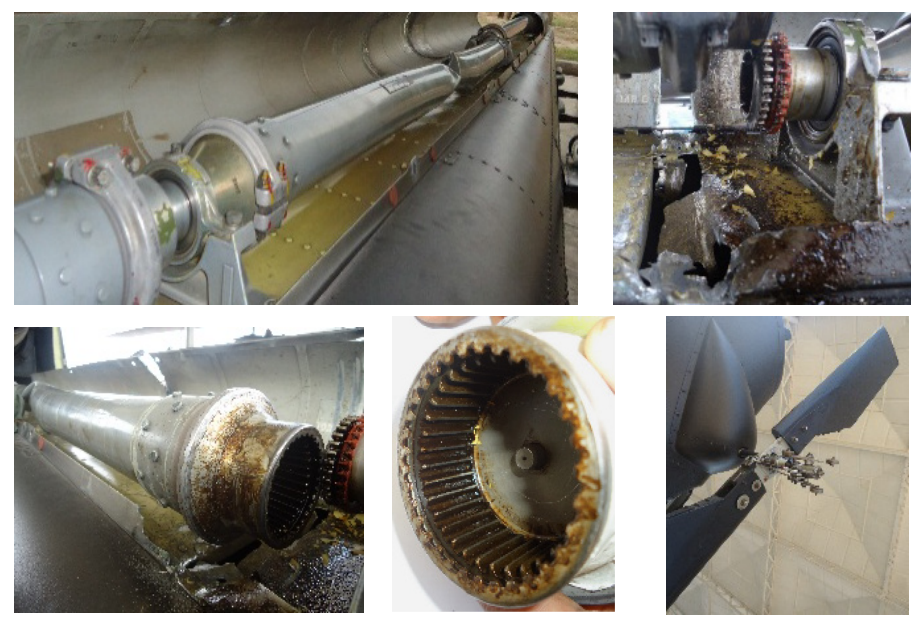

Fig. 2. Twisting of the tail rotor shaft and the damage done by the loose rotating shaft.

Stall \& Surge. A compressor stall describes a condition in which the flow of air in the compressor/turbine becomes unstable. The symptoms could be popping / roaring noise from the compressor, rising of combustion chamber temperature and loss of power depending upon the engine type and severity of stall. The compressor stall which lowers to power output is generally followed by a power surge when the normal / required air flow is restored.

Typically, there are multistage axial compressors which feed a centrifugal compressor prior to combustion in order to ensure smooth and ample air supply. However, the air requirements change significantly as the RPM changes. The air requirement is low at low RPM, therefore, a part of the air is made to bleed before it enters centrifugal compressor, while at high RPM all the air may be necessary.

The nominal time period required for the actuation of a variable stator or a bleed is about 200 msec. This contrasts with the time period for the development of rotating stall and surge which is on the order of several rotor revolutions. One rotor revolution for an aircraft gas turbine typically is about $5 \mathrm{msec}$, and can be as long as $20 \mathrm{msec}$ [2]. Other causes of compressor stall are damaged or dirty compressor blades, recirculation of hot exhaust gases being sucked into the compressor when hovering downwind, incorrectly set up fuel control units and poor intake design etc. [3].

With a single engine configuration, in general about $10 \%$ of the total power is transmitted to the tail rotor through the tail rotor shaft, thus a max of about $82 \mathrm{KW}$ is available for the concerned shaft to transmit. However, it was observed that there were five compressor stalls in last 30 operational hours. The accompanying power surge can possibly result in conditions similar to impact / high strain rate loading and thus can cause fracture without plastic deformation in otherwise ductile alloy.

Objective \& Methodolgy. The key objective of the present study were the followings:

- To inspect the structural material of the tail rotor shaft for its chemical composition, microstructure and mechanical properties.

To study the deformation and fracture mode of the shaft.

To determine the possible loading conditions that can cause the observed deformation and fracture.

$\square$ To determine the root cause of the incident.

- To determine whether this shaft twist occurred in air or during ground hitting.

In order to achieve the above mentioned objectives, the chemical analysis through ICP-OES was made on the representative samples of the shaft. The microstructure and the fractographic analysis were carried out using optical microscope and scanning electron microscope, respectively. The Rockwell hardness tester was used for the hardness testing. A FEM based simulation was performed on ANSYS to observe the equivalent stress in the shaft if one end of the shaft is given torque while keeping the other end fixed. The findings were linked together to fix the root cause of the incident. 


\section{Analytical Results}

The results of chemical analysis given in Table 1 matches with the aluminum alloy 2024 which is a common materials for the aerospace applications. The mechanical properties of the alloys (Table 2) show quite high yield stress and ultimate tensile stress values of $350 \mathrm{MPa}$ and $475 \mathrm{MPa}$, respectively with good elongation before fracture. Such values are common for any of the heat treated /tempered conditions i.e. T3, T4, T6 of the $2024 \mathrm{Al}$ alloy. The observed hardness and tensile strength values are almost similar which indicate the absence of any strain hardening which can happen after plastic deformation. The optical micrographs (Fig. 3) also show no evidence of any recrystallization which can happen during significant strain hardening. Thus the mechanical stress by large did not exceed the yield stress in the twisted regions, except of course at the cones of twisted ends where the material has fractured.

Table 1. Chemical analysis determined through ICP-OES analysis.

\begin{tabular}{|l|l|l|l|l|l|}
\hline $\mathbf{C u}$ & $\mathbf{M g}$ & $\mathbf{M n}$ & $\mathbf{F e}$ & $\mathbf{S i}$ & $\mathbf{A l}$ \\
\hline 4.42 & 1.43 & 0.40 & 0.12 & 0.15 & Bal. \\
\hline
\end{tabular}

Table 2. Hardness and tensile properties of the alloy.

\begin{tabular}{|l|l|l|}
\hline & Sound Part & Twisted Part \\
\hline Hardness & $63 \mathrm{HRB}$ & $65 \mathrm{HRB}$ \\
\hline Tensile yield stress & $370 \mathrm{MPa}$ & $375 \mathrm{MPa}$ \\
\hline Ultimate tensile stress & $475 \mathrm{MPa}$ & $470 \mathrm{MPa}$ \\
\hline Elongation before fracture & $20 \%$ & $19 \%$ \\
\hline
\end{tabular}
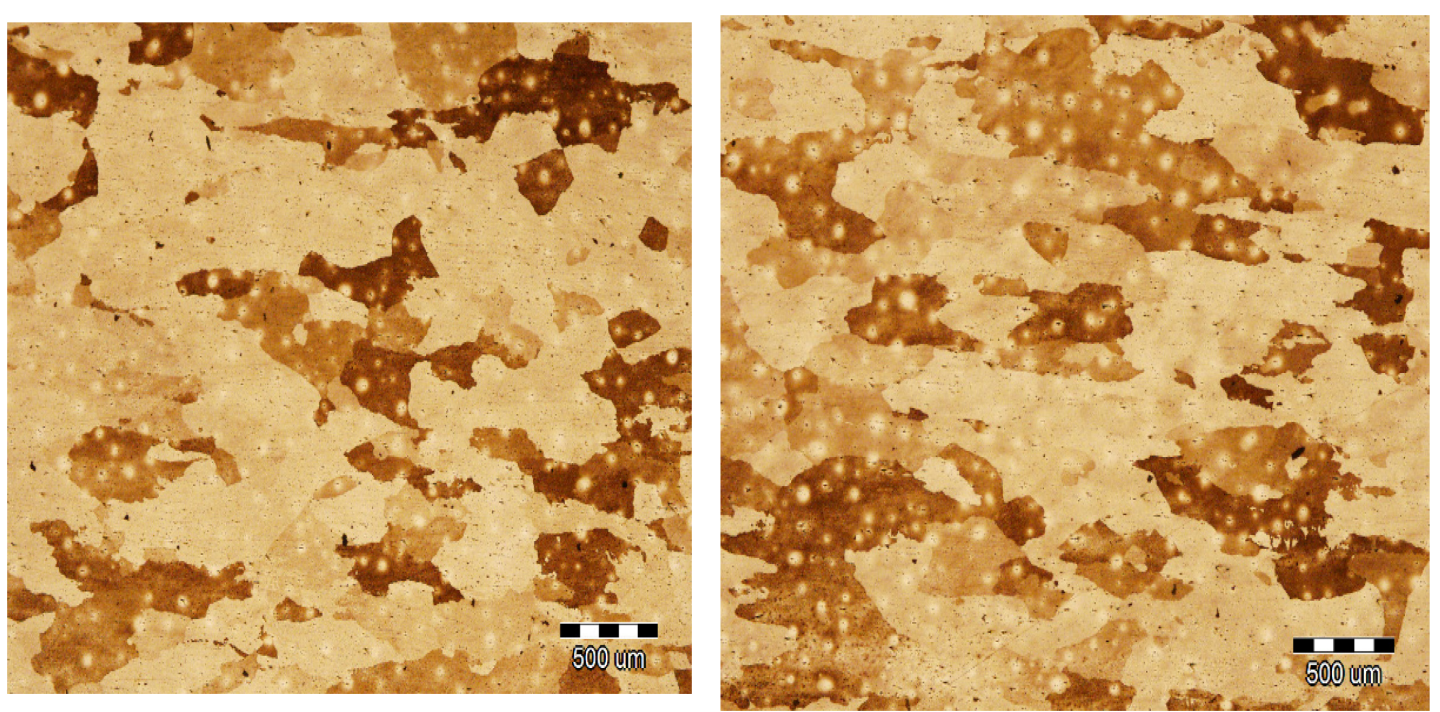

Fig. 3. Optical micrographs of (L) twisted and (R) sound sections of the shaft. 

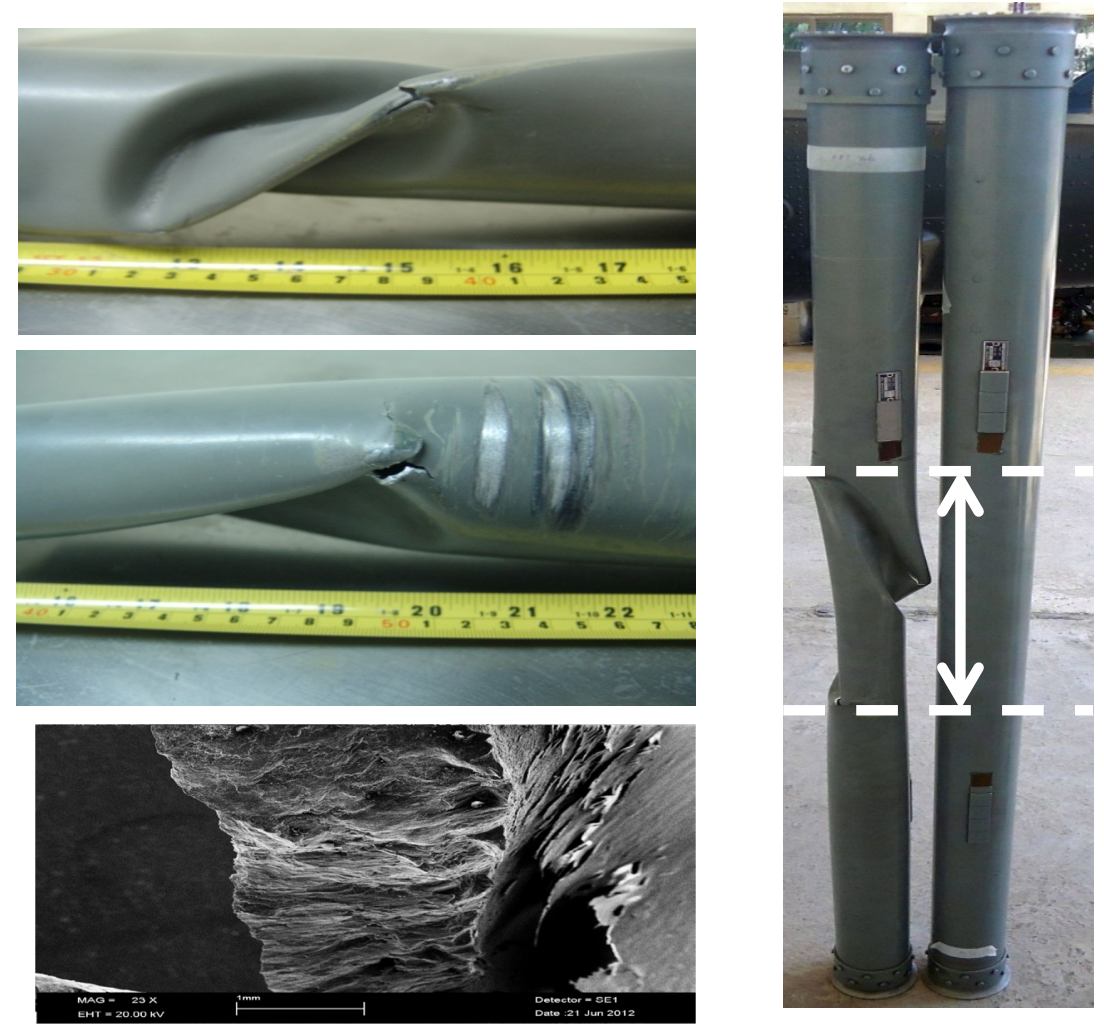

Fig. 4. Length shortening due to twisting in 14-inches section and shear fractures at twist ends.

The length of the twisted part 14 inches is about $25 \%$ of the total length (about 57 inch). The location of the twist is near center of the shaft. The twist caused length shortening of 0.7 inch which was enough to detach the shaft from the drive train connector at the farther end. The amount of damage shown in Fig. 2 clearly indicates that the shaft remained rotating after becoming free for quite some time.

There are fractures at three locations, i.e. at two twist ends and in the middle of twisted region. The camera images of the fractured regions show no evidence of plastic deformation before fracture (Fig. 4). The SEM fractographs also show no evidence of plastic deformation and show brittle fracture features. Moreover, no fatigue striations are present which rule out fatigue failure.
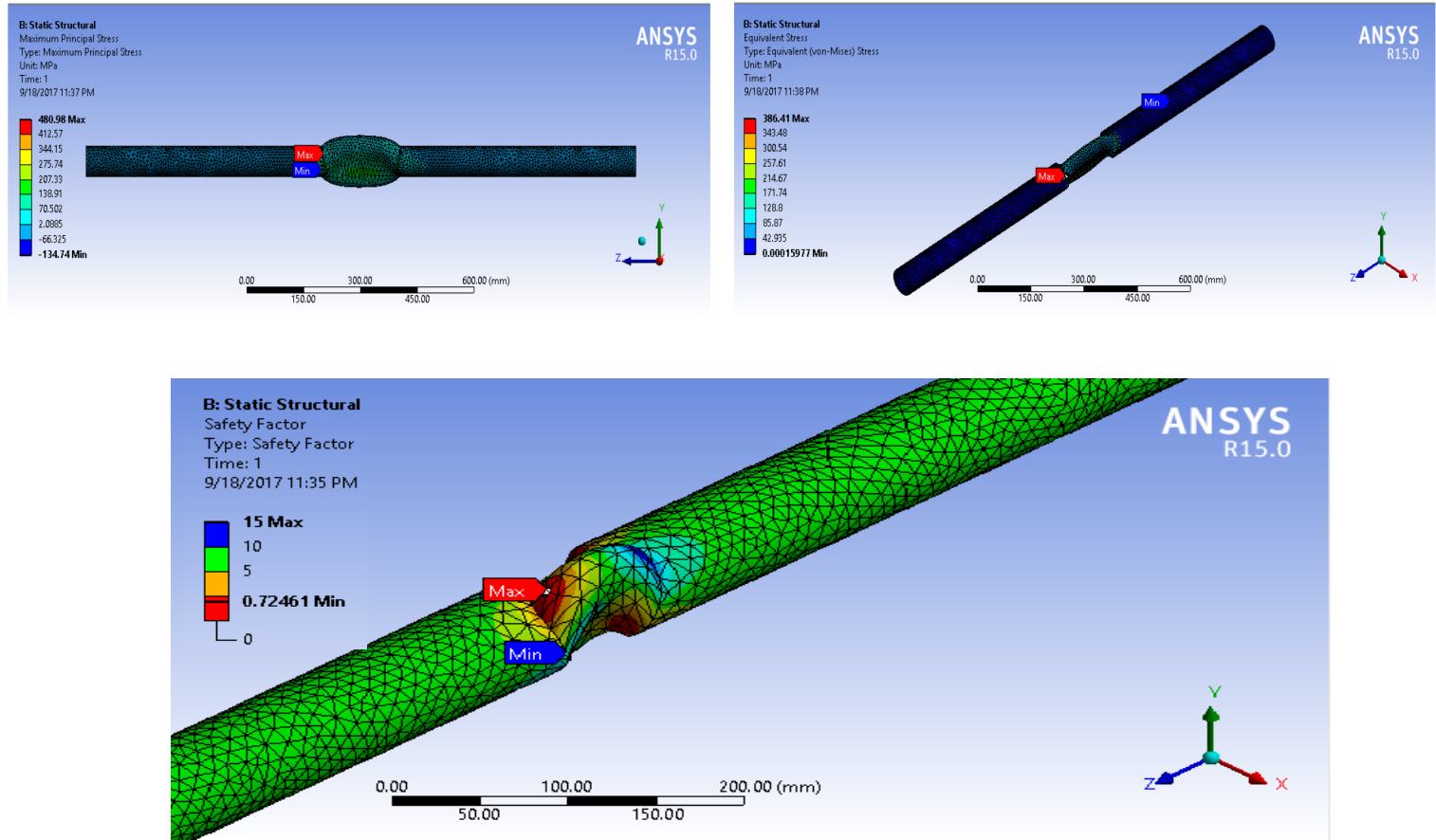

Fig. 5. ANSYS simulation showing stress concentration sites where the stress exceeds the UTS value. 
The FEM based analysis was carried out using commercial software ANSYS R15.0 to simulate the twist phenomenon by fixing one end of the shaft while applying torque at the other end. Several configurations were tried to simulate the observed twist that involves flattening of radial shaft with stress concentration sites at the twist ends. The closest results were obtained by restricting the z-axis movement and applying twist at selected y-z planes rather than at the shaft ends.

The ANSYS result proved two points. One, that twist cannot happen unless there is one end fixed. Thus, if shaft would have become free after ground hitting, the observed twist cannot occur. Second, that the twist can cause stress concentration in excess of the UTS at locations of shaft-twist intersection, while the stress at other location is below the yield stress, or even compressive at some locations.

The recheck showed that there was no blockage to the shaft rotation which could have caused the twist. The literature [4] shows that the high surge in power which cannot be transmitted by the shaft can create similar loading conditions leading to the down fall of the helicopter.

\section{Discussion \& Conclusions}

The results of chemical analysis, mechanical tests and microstructural analysis show that the shaft is made up of an Al-Cu-Mg alloy 2024. The mechanical properties of the shaft, at locations away from the twist as well as within the twist (the flattened part) conform to the standard values of the alloy in T3 condition. So material fault / malfunction is ruled out. The ANSYS simulations showed that twisting can cause the stress to exceed the UTS of the alloy at stress concentration sites, which is the reason for no observable plastic deformation before fracture in, otherwise, ductile materials is high during excessive strain rates. The twisting and fracture at stress concentration sites require quite high stress. To develop this much stress, the other end of the shaft needs support which is not possible if the shaft would become free under the ground hitting impact. Moreover, the twisted shaft in such case would not be as straight as observed in this case. Therefore, it is concluded that the observed shaft twist cannot happen due to the ground hitting impact. The stall and surge phenomenon stands out as most probable source of high strain rate shaft rotation. It was verified that the helicopter had observed five stalls in last 30 operation hours and there was some observable stall related damage to the turbine blades as well. Thus, compressor stall and subsequent power surge is concluded as the root cause.

\section{References}

[1] What is the function of a tail rotor shaft in a helicopter? https://www.quora.com/What-is-thefunction-of-the-tail-rotor-on-a-helicopter ,2018 (accessed 7th Feburary 2018).

[2] L.S. Langston, Gas-turbine compressors: Understanding stall, surge, Combined Cycle Journal, http://www.ccj-online.com/combined-cycle-journal-number-50/gas-turbine-compressorsunderstanding-stall-surge ,2018 (accessed 9th Feburary 2018).

[3] Surge and compressor stall, http://www.pprune.org/rotorheads/68907-surge-compressorstall.html ,2018 (accessed 5th Feburary 2018).

[4] Patrick Veillette Jumprsaway, Compressor stalls in turbine helicopters, Aviation Week, http://aviationweek.com/awin/compressor-stalls-turbine-helicopters ,2018 (accessed 9th Feburary 2018) 